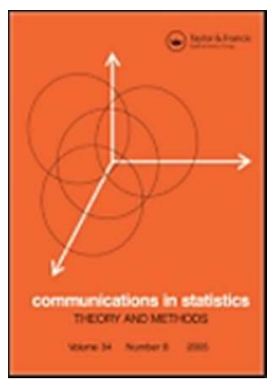

\title{
Balancing desirability and promotion steadiness in partially stochastic manpower planning systems
}

\begin{tabular}{|r|l|}
\hline Journal: & Communications in Statistics - Theory and Methods \\
\hline Manuscript ID: & LSTA-2013-0896.R1 \\
\hline Manuscript Type: & Special Issue - ASMDA2013 \\
\hline Date Submitted by the Author: & $\mathrm{n}$ a \\
\hline Keywords: & $\begin{array}{l}\text { List of Authors: } \\
\text { komarudin, komarudin; Vrije Universiteit Brussel, BUTO } \\
\text { De Feyter, Tim; KU Leuven, Department of Business Management Research } \\
\text { Vanden Berghe, Greet; KU Leuven, Department of Computer Science }\end{array}$ \\
\hline Abstract: & $\begin{array}{l}\text { Current manpower planning approaches focus on satisfying future } \\
\text { personnel needs. In the mean time, there is a lack of contribution on } \\
\text { managing the personnel work satisfaction on the long-term. } \\
\text { In general, long-term work satisfaction can be achieved by maintaining the } \\
\text { preferred promotion strategy. This paper aims at balancing the strategy to } \\
\text { fulfill the future personnel requirements while at the same time } \\
\text { maintaining the preferred promotion strategy. Moreover, stochastic } \\
\text { wastage is considered to better capture the real world condition of } \\
\text { voluntarily wastage. Illustration of the model shows that it enables } \\
\text { obtaining favorable recruitment and promotion strategies. }\end{array}$ \\
\hline
\end{tabular}

\section{SCHOLARONE ${ }^{\text {M }}$ \\ Manuscripts}




\title{
Balancing desirability and promotion steadiness in
}

\section{partially stochastic manpower planning systems}

\author{
Komarudin*1, Tim De Feyter ${ }^{\dagger 2}$, Marie-Anne Guerry ${ }^{\ddagger 1}$, and Greet Vanden \\ Berghe $^{\S 3}$
}

${ }^{1}$ Vrije Universiteit Brussel, Department of Business Technology and Operations

${ }^{2} \mathrm{KU}$ Leuven, Department of Business Management Research ${ }^{3}$ KU Leuven, Department of Computer Science

23 June 2014

\begin{abstract}
Current manpower planning approaches focus on satisfying future personnel needs. In the mean time, there is a lack of contribution on managing the personnel work satisfaction on the long-term. In general, long-term work satisfaction can be achieved by maintaining the preferred promotion strategy. This paper aims at balancing the strategy to fulfill the future personnel requirements while at the same time maintaining the preferred promotion strategy. Moreover, stochastic wastage is considered to better capture the real world condition of voluntarily wastage. Illustration of the model shows that it enables obtaining favorable recruitment and promotion strategies.

*Email: komarudin@vub.ac. be

${ }^{\dagger}$ Email: tim.defeyter@kuleuven.be

†Email: maguerry@vub.ac.be

${ }^{\S}$ Email: greet.vandenberghe@cs.kuleuven.be
\end{abstract}


Keywords: manpower planning, Markov model, fuzzy sets, stochastic wastage.

\section{Introduction}

Manpower planning is concerned with fulfilling the organization's personnel requirements, in order to support the long-term organizational strategy. Several statistical techniques based on Markov's theory have been developed to support organizations in this challenging human resource management process (Bartholomew et al., 1991). In general, these methods require the employees to be classified in exclusive subgroups according to personal and/or work-related characteristics (De Feyter, 2006). The number of people in each of the subgroups is represented by a personnel structure. The main objective of manpower planning is to attain or maintain the desired personnel structure. To tackle this problem, the evolution of the personnel structure needs to be examined and, if necessary, adjusted towards the direction of the desired personnel structure. In a manpower model, the evolution of a personnel structure is determined by employee transitions, i.e. recruitments, promotions (i.e. employee flows between the employee subgroups) and wastage.

Early academic contributions in manpower planning already studied the attainability problem for deterministic personnel systems, in which the model parameters are known. In these models, the evolution of personnel structures is regulated by known employee transitions, which may or may not be under management control. Several methods were developed to support organizations in attaining desired personnel structures under control by recruitment and/or promotion. In real-world applications, however, the deterministic assumptions rarely hold, because manpower planning is subject to uncertainty about the model parameters. Therefore, other advances in the field place emphasis on stochastic manpower planning models. Firstly, a number of scholars recently focus on uncertain personnel requirements. Zhu and Sherali (2009), for example, propose a mixed integer formulation for a manpower planning problem with uncertain personnel demand fluctuations. Sohoni et al. (2004), on the other hand, propose an optimization strategy to 
estimate long-range crew staffing considering randomness due to operational disruptions. Secondly, another research stream in manpower planning considers uncertainty about employee transition processes. Chattopadhyay and Gupta (2007), for example, studied a stochastic manpower planning problem with random age-based survival rates. Indeed, voluntary wastage, in particular, is likely to be uncertain and highly beyond management control. Therefore, the attainability problem was often studied for the partially stochastic manpower planning model, in which wastage is treated as a random variable and promotion rates are known.

Previous work studied the probability of attaining future personnel structures in partially stochastic manpower planning systems under control by recruitment Guerry, 1993; Davies, 1982). It appears that in such models the desired personnel structures are not always attainable. Several approaches to this problem are possible. De Feyter and Guerry (2009) suggest a fuzzy based method for evaluating a recruitment strategy in a stochastic manpower planning system based on the degree of desirability. This measure reflects the extent to which the resulting personnel structure is similar to the unattainable desired one. Although this method ensures that the personnel structure evolves in the direction of the desired one, it does not attain it. An alternative approach could be to extend the decision variables in the manpower planning model beyond recruitment only. Song and Huang (2008), Nilakantan et al. (2011) and Dimitriou et al. (2013), for example, propose methods that intend to fulfill the personnel requirements without explicitly maintaining the promotion strategy". This approach does allow to attain the desired personnel structure. But, in order to meet the personnel requirements, it may be inevitable to adjust the promotion strategy. This is a considerable disadvantage because changing promotion opportunities and uncertainty about career progression may harm employees' job satisfaction (Klehe et al., 2011). Consequently, organizations potentially are confronted with a trade-off problem between attainability of a desired personnel structure and maintainability of the promotion strategy.

In the present paper, we propose an approach to this trade-off problem in partially stochastic manpower systems. Our method supports organizations in balancing two po- 
tentially conflicting objectives.

In particular, we consider the objectives of attaining the personnel requirements and maintaining a promotion strategy. In Section 2, we present a deterministic manpower planning model that describes the attainability problem under control by recruitment and promotion. Besides control by recruitment, modifications of the promotion strategy are allowed. We consider two criteria for evaluating the recruitment and promotion strategies. On the one hand, as in De Feyter and Guerry (2009), we want to maximize the degree of desirability. In this way, the long-term organizational strategy can be supported in the best possible way. On the other hand, we introduce the degree of promotion steadiness that represents to what extent the performed promotions correspond with the preferred promotion strategy. Since the preferred promotion strategy leads to clear career progression and career prospects for employees, we want to maintain the preferred promotion probabilities. In this way, the long-term personnel work satisfaction is maintained.

In Section 3, we transform the deterministic model from Section 2 into a partially stochastic model. We propose the DPS problem (desirability and promotion steadiness problem) that is balancing the degree of desirability and the degree of promotion steadiness for partially stochastic manpower systems. The stochastic wastage is approximated with a sufficient number of possible wastage scenarios and hence, the problem can be solved with MIP solver. However, as we will show, the mixed integer programming (MIP) model is NP-hard. Consequently, an increase in the number of subgroups is likely to lead to an exponential increase in computing time. In Section 4, we discuss and illustrate how our model and proposed algorithm can be applied and which insights could be employed in a practical context. Finally, some conclusions and suggestions for further research are discussed in Section 5 , 


\section{Desirability and promotion steadiness in a deter- ministic model}

Assume the personnel is categorized into $k$ subgroups, i.e. $\left\{v_{1}, v_{2}, \ldots, v_{k}\right\}$. Let us denote $n_{i}^{t}$ the number of personnel of subgroup $v_{i}$ at time $t$. The personnel structure $n^{t}$ at time $t$ is the vector with elements $n_{i}^{t}$. $N^{\text {min }}$ and $N^{\max }$ refer to the lower and the upper bound of the total number of personnel. Those bounds can be induced by e.g. capacity or budget constraints. A person from subgroup $v_{i}$ can become a member of subgroup $v_{j}$ based on the transition proportion $p_{i j}$ after one period of time. $p_{i j}$ can be gathered into the internal transition matrix $\mathbf{P}$. The personnel flows $f_{i j}^{t-1, t}$ denote the number of personnel in subgroup $v_{i}$ at time $t-1$ that become members of subgroup $v_{j}$ at time $t$, and $\mathbf{F}^{\mathbf{t}-\mathbf{1 , t}}$ represents the flow matrix with elements $f_{i j}^{t-1, t}$.

We denote $R_{i}^{t}$ for the number of personnel recruited to subgroup $v_{i}$ at time $t$, and $\mathbf{R}^{\mathbf{t}}$ for the recruitment vector at time $t$. Then, the evolution of the personnel structure from $t-1$ to $t$ is expressed by Eq. 1 (Bartholomew et al., 1991). It is assumed that $R_{i}^{t}$ is non-negative. The personnel wastage $W_{i}^{t-1, t}$ expresses the number of personnel in subgroup $v_{i}$ at time $t-1$ that no longer belong to any subgroup at time $v_{t}$. The wastage proportion for subgroup $v_{i}$ is $w_{i}$. Then, the relation between the elements of $\mathbf{P}$ and $\mathbf{w}$ can be described as in Eq. 2.

$$
\begin{gathered}
\mathbf{n}^{\mathbf{t}}=\mathbf{n}^{\mathbf{t}-\mathbf{1}} \mathbf{P}+\mathbf{R}^{\mathbf{t}} \\
\sum_{j=1}^{k} p_{i j}+w_{i}=1
\end{gathered}
$$

The objective of the deterministic model is to obtain the best recruitment vector $\mathbf{R}^{\mathbf{t}}$ and personnel flows $\mathbf{F}^{\mathbf{t}-\mathbf{1 , t}}$ considering the period from $t-1$ to $t$. Two criteria are considered, i.e. the degree of desirability $\beta\left(\mathbf{n}^{\mathbf{t}}\right)$ and the degree of promotion steadiness $\gamma\left(\mathbf{F}^{\mathbf{t}-1, \mathbf{t}}\right)$.

The degree of desirability of $\mathbf{n}^{\mathbf{t}}$ is reflected by the discrepancy between the personnel 
structure $\mathbf{n}^{\mathbf{t}}$ and the desired personnel structure $\mathbf{n}^{\mathbf{d}}$. The discrepancy between $\mathbf{n}^{\mathbf{t}}$ and $\mathbf{n}^{\mathbf{d}}$ should be minimized because $\mathbf{n}^{\mathbf{d}}$ reflects the personnel structure that supports the organizational long-term goals. De Feyter and Guerry (2009) proposed a fuzzy set approach for defining the degree of desirability. Eq. 3 denotes that the desirability degree formula uses the fuzzy min-operator. Let $n_{i}^{L L}$ and $n_{i}^{U L}$ be the lower and upper limit of the number of personnel of subgroup $v_{i}$. The fuzzy triangular function $\beta_{i}\left(n_{i}^{t}\right)$ as in Eq. 4 states how far the number of personnel of subgroup $v_{i}$ deviates from the desired number of personnel $n_{i}^{d}$. Other fuzzy membership functions may be alternatives for defining $\beta\left(\mathbf{n}^{\mathbf{t}}\right)$.

$$
\begin{gathered}
\beta\left(\mathbf{n}^{\mathbf{t}}\right)=\min _{i} \beta_{i}\left(n_{i}^{t}\right) \\
\beta_{i}\left(n_{i}^{t}\right)= \begin{cases}0, & \text { if } n_{i}^{t}<n_{i}^{L L} \text { or } n_{i}^{t}>n_{i}^{U L} \\
\frac{n_{i}^{t}-n_{i}^{L L}}{n_{i}^{d}-n_{i}^{L L}}, & \text { if } n_{i}^{L L} \leq n_{i}^{t} \leq n_{i}^{d} \\
\frac{n_{i}^{t}-n_{i}^{U L}}{n_{i}^{d}-n_{i}^{U L}}, & \text { if } n_{i}^{d} \leq n_{i}^{t} \leq n_{i}^{U L}\end{cases}
\end{gathered}
$$

We assume that intervention in the personnel flows $f_{i j}^{t-1, t}$ are possible. Nevertheless, large deviations from the internal transition matrix are not preferable. The degree of promotion steadiness is introduced to express to what extent the performed personnel flows correspond to the internal transition matrix. Let $\tilde{p}_{i j}$ the proportion $f_{i j}^{t-1, t} / n_{i}^{t-1}$ of the performed personnel flow from subgroup $v_{i}$ to subgroup $v_{j}$. Specifically, the degree of promotion steadiness $\gamma_{i j}\left(f_{i j}^{t-1, t}\right)$ measures the discrepancy between a personnel flow proportion $\tilde{p}_{i j}$ and its corresponding transition proportion $p_{i j}$ (i.e. the $i j$ th element of the transition matrix $\mathbf{P}$ ). The discrepancy should be minimized so that the organization's intervention in personnel promotion can be suppressed. Analogue to the degree of desirability, we express the degree of promotion steadiness by a triangular fuzzy number. Eq. 5 denotes that the promotion steadiness degree formula uses the fuzzy min-operator. Eq. 6 uses a fuzzy triangular function with lower limit $p_{i j}^{L L}$ and upper limit $p_{i j}^{U L}$ for defining $\gamma_{i j}\left(f_{i j}^{t-1, t}\right)$. If the proportion $f_{i j}^{t-1, t} / n_{i}^{t-1}$ is between the lower limit $p_{i j}^{L L}$ and upper limit $p_{i j}^{U L}$, then its corresponding desirability degree is determined by how far it deviates from the transition proportion $p_{i j}$. This is stated in Eq. 6. The lower limit and the upper 
limit of the triangular membership function related to $\tilde{p}_{i j}$ can be set to negative numbers although the membership function is only considered for $\tilde{p}_{i j} \in[0,1]$. We can also use other fuzzy membership functions for defining $\gamma_{i j}\left(f_{i j}^{t-1, t}\right)$.

$$
\begin{gathered}
\gamma\left(\mathbf{F}^{\mathbf{t - 1 , \mathbf { t }}}\right)=\min _{i, j} \gamma_{i j}\left(f_{i j}^{t-1, t}\right) \\
\gamma_{i j}\left(f_{i j}^{t-1, t}\right)= \begin{cases}0, & \text { if } \tilde{p}_{i j}<p_{i j}^{L L} \text { or } \tilde{p}_{i j}>p_{i j}^{U L} \\
\frac{\tilde{p}_{i j}-p_{i j}^{L L}}{p_{i j}-p_{i j}^{L L}}, & \text { if } p_{i j}^{L L} \leq \tilde{p}_{i j} \leq p_{i j} \\
\frac{\tilde{p}_{i j}-p_{i j}^{U L}}{p_{i j}-p_{i j}^{U L}}, & \text { if } p_{i j} \leq \tilde{p}_{i j} \leq p_{i j}^{U L}\end{cases}
\end{gathered}
$$

Let the overall degree $\kappa\left(\mathbf{n}^{\mathbf{t}}, \mathbf{F}^{\mathbf{t}-\mathbf{1 , t}}\right)$ incorporates both the degree of desirability and the degree of promotion steadiness. We consider a min-operator when maximizing the overall degree $\kappa\left(\mathbf{n}^{\mathbf{t}}, \mathbf{F}^{\mathbf{t}-\mathbf{1}, \mathbf{t}}\right)$ that is represented in Eq. 7 .

$$
\begin{aligned}
& \operatorname{maximize} \kappa\left(\mathbf{n}^{\mathbf{t}}, \mathbf{F}^{\mathbf{t}-\mathbf{1}, \mathbf{t}}\right) \\
& \text { with } \kappa\left(\mathbf{n}^{\mathbf{t}}, \mathbf{F}^{\mathbf{t}-\mathbf{1 , t}}\right)=\min \left\{\beta\left(\mathbf{n}^{\mathbf{t}}\right), \gamma\left(\mathbf{F}^{\mathbf{t}-\mathbf{1 , t}}\right)\right\}
\end{aligned}
$$

with $\min \left\{\beta\left(\mathbf{n}^{\mathbf{t}}\right), \gamma\left(\mathbf{F}^{\mathbf{t}-\mathbf{1}, \mathbf{t}}\right)\right\}$ a min-operator that selects the minimum of the degree of desirability $\beta\left(\mathbf{n}^{\mathbf{t}}\right)$ and the degree of promotion steadiness $\gamma\left(\mathbf{F}^{\mathbf{t - 1 , t}}\right)$. The max-min approach is selected in order to guarantee that both $\beta\left(\mathbf{n}^{\mathbf{t}}\right)$ and $\gamma\left(\mathbf{F}^{\mathbf{t}-\mathbf{1}, \mathbf{t}}\right)$ are as large as possible.

\section{Desirability and promotion steadiness in a par- tially stochastic model}

In this section, we transform the deterministic model from Section 2 into a partially stochastic model. Afterwards, a Mixed Integer Programming (MIP) is proposed to optimize the degree of desirability and the degree of promotion steadiness. The model can be solved by an existing method, i.e. branch and bound algorithm.

A model that fits the wastage observations can be achieved when considering stochastic wastage, as was already shown by (Agrafiotis, 1984). By using stochastic wastage, the organization can develop personnel strategies that are able to deal with unexpected 
wastage levels. In particular, we assume that the recruitment strategy must be determined before the personnel wastage is known.

The wastage proportion $w_{i}$ is assumed to be a random variable. The $i$ th row sum of the internal transition matrix $\mathbf{P}$ depends on $w_{i}$ (as in Eq. 2). Since the admission and the training process of new employees can take a significant amount of time, the recruitment strategy should be chosen before the realization of $\mathbf{w}$ is known. Meanwhile, the selection of the promotion strategy is postponed until $w$ is known. As a result, the model aims at obtaining a robust recruitment vector $\mathbf{R}^{\mathbf{t}}$ and flow matrix $\mathbf{F}^{\mathbf{t}-\mathbf{1}, \mathbf{t}}$ that can maximize both the degree of desirability and the degree of promotion steadiness. Note that the model results in attainable personnel structures since the recruitment vector $\mathbf{R}^{\mathbf{t}}$ is assumed to be non-negative.

In most practical applications, the random variables are approximated by a finite number of scenarios (Shapiro et al. 2009). We consider $S$ scenarios for the random variable $w$, i.e. $\left\{\tilde{w}^{1}, . ., \tilde{w}^{S}\right\}$. The recruitment vector $R^{t}$ will be the same for each scenario because it is determined before the realization of the wastage. Let $\mathbf{n}^{\mathbf{t}, \mathbf{s}}$ and $\mathbf{F}^{\mathbf{t}-\mathbf{1 , t}, \mathbf{s}}$ be a feasible personnel structure and flow matrix under scenario $s$. The average overall degree $\bar{\kappa}$ is approximated by the average of $\left\{\kappa\left(\mathbf{n}^{\mathbf{t}, \mathbf{1}}, \mathbf{F}^{\mathbf{t}-\mathbf{1}, \mathbf{t}, \mathbf{1}}\right) \ldots, \kappa\left(\mathbf{n}^{\mathbf{t}, \mathbf{S}}, \mathbf{F}^{\mathbf{t}-\mathbf{1}, \mathbf{t}, \mathbf{S}}\right)\right\}$ resulting from each scenario.

In previous work, the total number of personnel is often considered to be constant (see e.g. Guerry and De Feyter (2012)). In the present model, it might not be possible to accommodate this constraint, since the scenarios may result in personnel structures with different total number of personnel. However, the total number of personnel is restricted by the lower bound $N^{\min }$ and the upper bound $N^{\max }$. The model also can accommodate additional constraints, such as maximum or minimum value for $n_{i}^{t}$.

\subsection{Mathematical formulation}

The problem of balancing the degree of desirability and the degree of promotion steadiness for partially stochastic manpower systems is called the DPS problem (desirability and promotion steadiness problem). We formulate the DPS problem as a mixed integer 
program (MIP) (Eqs. 825). Eq. 8 expresses the objective to maximize the average of the overall degree for the different scenarios $s \in\{1,2, \ldots, S\}$.

The presented model corresponds to the triangular fuzzy membership functions defined in Eqs. 4 and 6. In order to get the formulation of the MIP, the fuzzy triangular functions are converted into piecewise linear constraints (Eqs. 9. 18). The fuzzy triangular function $\beta_{i}\left(n_{i}^{t}\right)$ is partitioned into two segments, the left part corresponds to values $n_{i}^{t}$ that are less than $n_{i}^{d}$ and the right part corresponds to values $n_{i}^{t}$ that are greater than $n_{i}^{d}$. Similarly, the fuzzy triangular function $\gamma_{i j}\left(f_{i j}^{t-1, t}\right)$ is partitioned into a left and a right part, that correspond to values $\tilde{p}_{i j}$ respectively smaller and greater than $p_{i j}$. Let $q_{i L}^{s b 1}, q_{i R}^{s b 1}$, $q_{i j L}^{s b 2}$ and $q_{i j R}^{s b 2}$ be binary variables; $q_{i}^{s c 1}$ and $q_{i j}^{s c 2}$ be continuous variables between zero and one. The binary variables $q_{i L}^{s b 1}, q_{i R}^{s b 1}, q_{i j L}^{s b 2}$ and $q_{i j R}^{s b 2}$ are introduced to differentiate between the left and the right parts of the fuzzy triangular function. $q_{i L}^{s b 1}=1$ indicates that $n_{i}^{t, s}$ is at the left of $n_{i}^{d}$ while $q_{i R}^{s b 1}=1$ indicates that $n_{i}^{t, s}$ is at right side of $n_{i}^{d}$. Similarly, $q_{i j L}^{s b 2}=1$ indicates that $\tilde{p}_{i j}^{s}$ is at the left of $p_{i j}$ while $q_{i j R}^{s b 2}=1$ indicates that $\tilde{p}_{i j}^{s}$ is at right side of $p_{i j}$. Continuous variables $q_{i}^{s c 1}\left(q_{i j}^{s c 2}\right)$ are used in Eqs. 13 (Eqs. 18 to define $n_{i}^{t, s}\left(\tilde{p}_{i j}^{s}\right)$ as a convex combination of $n_{i}^{L L}, n_{i}^{d}$ and $n_{i}^{U L}\left(p_{i j}^{L L}, p_{i j}\right.$ and $\left.p_{i j}^{U L}\right)$.

Eq. 19 calculates the performed personnel flow $f_{i j}^{t-1, t, s}$ corresponding to $\tilde{p}_{i j}^{s}$. The value of $\tilde{\kappa}^{s}\left(n^{t, s}, F^{t-1, t, s}\right)$ is only determined by the coefficients $q_{i 2}^{s c 1}$ and $q_{i j 2}^{s c 2}$ (Eq. 20.21). Eq. 22 restricts the total personnel in the system by the lower bound $N^{\text {min }}$ and the upper bound $N^{\max }$. Eq. 23 specifies that the personnel of a subgroup originates from the personnel flows into the subgroups and the personnel recruitment into that subgroup. Eq. 24 indicates that personnel of a subgroup can remain in the subgroup, move to other subgroups or move out of the organization.

As suggested by Guerry (2008) to enhance the accuracy of the model, we require $n_{i}^{t, s}$, $f_{i j}^{t-1, t, s}, R_{i}^{t}$ to be integer numbers. Eq. 25 expresses the integrality and nonnegativity constraints of $n_{i}^{t, s}, f_{i j}^{t-1, t, s}, R_{i}^{t}$.

$$
\operatorname{maximize} \bar{\kappa}=\frac{1}{S} \sum_{s=1}^{S}\left(\kappa\left(n^{t, s}, F^{t-1, t, s}\right)\right)
$$




$$
\begin{aligned}
& q_{s i 1}^{c 1} \leq q_{i L}^{s b 1} \quad \forall s, i \\
& q_{i 3}^{s c 1} \leq q_{i R}^{s b 1} \quad \forall s, i \\
& q_{i L}^{s b 1}+q_{i R}^{s b 1}=1 \quad \forall s, i \\
& \sum_{j=1}^{3} q_{i j}^{s c 1}=1 \quad \forall s, i \\
& n_{i}^{t, s}=q_{i 1}^{s c 1} n_{i}^{L L}+q_{i 2}^{s c 1} n^{d}+q_{i 3}^{s c 1} n_{i}^{U L} \quad \forall s, i \\
& q_{i j 1}^{s c 2} \leq q_{i j L}^{s b 2} \quad \forall s, i, j \\
& q_{i j 3}^{s c 2} \leq q_{i j R}^{s b 2} \quad \forall s, i, j \\
& q_{i j L}^{s b 2}+q_{i j R}^{s b 2}=1 \quad \forall s, i, j \\
& \sum_{k=1}^{3} q_{i j k}^{s c 2}=1 \quad \forall s, i, j \\
& \tilde{p}_{i j}^{s}=q_{i j 1}^{s c 2} p_{i j}^{L L}+q_{i j 2}^{s c 2} p_{i j}+q_{i j 3}^{s c 2} p_{i j}^{U L} \quad \forall s, i, j \\
& f_{i j}^{t-1, t, s}=\tilde{p}_{i j}^{s} n_{i}^{t-1} \quad \forall s, i, j \\
& \kappa\left(n^{t, s}, F^{t-1, t, s}\right) \leq q_{i 2}^{s c 1} \quad \forall s, i
\end{aligned}
$$




$$
\kappa\left(n^{t, s}, F^{t-1, t, s}\right) \leq q_{i j 2}^{s c 2} \quad \forall s, i, j
$$

$$
N^{\min } \leq \sum_{i=1}^{k} n_{i}^{t, s} \leq N^{\max } \forall s
$$

$$
n_{i}^{t, s}=\sum_{j=1}^{k} f_{j i}^{t-1, t, s}+R_{i}^{t} \quad \forall s, i
$$

$$
n_{i}^{t-1}=\sum_{j=1}^{k} f_{i j}^{t-1, t, s}+\pi_{i}^{t-1, t, s} \quad \forall s, i
$$

$$
n_{i}^{t, s}, f_{i j}^{t-1, t, s}, R_{i}^{t} \in \mathbb{Z}^{+} ; q_{i}^{s c 1}, q_{i j}^{s c 2}, \tilde{p}_{i j}^{s} \in[0,1] ; q_{i L}^{s b 1}, q_{i R}^{s b 1}, q_{i j L}^{s b 2}, q_{i j R}^{s b 2} \in\{0,1\}
$$

\subsection{Complexity of the DPS problem}

We continue with a discussion on the complexity of DPS problem. DPS can be viewed as a set of $S$ directed weighted graphs $G^{s}=(V, E)$. For each scenario $s, G^{s}$ has the same set $V$ of vertices. For time $t-1$, let us introduce $V^{t-1}=\left\{v_{i}^{t-1} \mid i \in\{1, . . k\}\right\}$ with vertex $v_{i}^{t-1}$ representing subgroup $v_{i}$. In the same way, for time $t, V^{t}=\left\{v_{i}^{t} \mid i \in\{1, . . k\}\right\}$ with vertex $v_{i}^{t}$ representing subgroup $v_{i}$. Let $V^{t-1}=\left\{v_{i}^{t-1} \mid i \in\{1, . . k\}\right\}$ and $V^{t}=\left\{v_{i}^{t} \mid i \in\{1, . . k\}\right\}$ with vertex $v_{i}^{t-1}$ and $v_{i}^{t}$ representing subgroup $v_{i}$. We provide two additional vertices, the source vertex $v_{0}$ and the sink vertex $v_{k+1}$. The set of vertices of the graph $G^{s}$ is defined by $V=V^{t-1} \cup V^{t} \cup\left\{v_{0}, v_{k+1}\right\}$. Let the set of edges $E$ of graph $G^{s}$ be defined by $E=\left\{\left(v_{i}^{t-1}, v_{j}^{t}\right) \mid \forall i, j \in\{1, . . k\}\right\} \cup\left\{\left(v_{0}, v_{i}^{t}\right) \mid \forall i \in\{1, . . k\}\right\} \cup\left\{\left(v_{i}^{t-1}, v_{k+1}\right) \mid \forall i \in\{1, . . k\}\right\}$. For the weighted graph $G^{s}$, let $n_{i}^{t-1}$ and $n_{i}^{t, s}$ be the capacity of $v_{i}^{t-1}$ and $v_{i}^{t}$. The personnel flow $f_{i j}^{t-1, t, s}$ is associated with edge $\left(v_{i}^{t-1}, v_{j}^{t}\right)$. The personnel wastage $\pi_{i}^{t-1, t, s}$ is associated with edge $\left(v_{i}^{t-1}, v_{k+1}\right)$. The recruitment $R_{i}^{t}$ is associated with edge $\left(v_{0}, v_{i}^{t}\right)$.

The parameter values of $n_{i}^{t-1}$ and $\pi_{i}^{t-1, t, s}$ are given. The problem is to find nonnegative values of $R_{i}^{t}$ (common for all $G^{s}$ ), $n_{i}^{t, s}$ and $f_{i j}^{t-1, t, s}$ that satisfy Eqs. 22, 24. The decision variables $n_{i}^{t, s}$ and $f_{i j}^{t-1, t, s}$ determine the objective function value of graph $G^{s}$ according 
to Eqs. 4, 6 and 7. The objective function value of DPS is the average of the objective function value of $G^{s}$.

We provide a theorem that describes the DPS problem to be NP-hard. The proof is based on a reduction of the 3-SAT into a partially-relaxed-DPS problem. The 3-SAT problem is stated as in Garey and Johnson (1979):

Definition 1. Given $C=\left\{c_{1}, c_{2}, \ldots, c_{l}\right\}$ of clauses on a finite set $U=\left\{u_{1}, u_{2}, \ldots, u_{m}\right\}$ of variables such that $\left|c_{h}\right|=3$ for $1 \leq h \leq l$. 3-SATISFIABILITY (3-SAT) is defined as a problem to decide whether there exists a truth assignment for $U$ that satisfies all the clauses in $C$.

The 3-SAT problem is a NP-complete problem (Garey and Johnson, 1979). A clause $c_{h}$ in the 3-SAT problem is a disjunction of three literals. A literal represents a single variable $u_{h}$ or its negation $\neg u_{h}$. Given $l$ clauses that involve $m$ variables, the 3 -SAT problem is satisfiable if there exists an assignment to the variables such that all the clauses are true.

Definition 2. A partially-relaxed-DPS is a relaxation of DPS in which the integrality constraint of some (but not all) variables $n_{s i}^{t}$ and $f_{s i j}^{t-1, t}$ is not imposed.

Lemma 1. The DPS and the partially-relaxed-DPS are in NP.

Proof. It is easy to see that DPS $\in$ NP and partially-relaxed-DPS $\in$ NP, since the objective value $\kappa\left(\mathbf{n}^{\mathbf{t}}, \mathbf{F}^{\mathbf{t}-\mathbf{1 , t}}\right)$ and satisfaction of constraints Eqs. 22,24 can be checked in polynomial time of size $k$ (the number of subgroups) and $S$ (the number of scenarios), given a DPS solution.

Lemma 2. If the partially-relaxed-DPS is NP-hard, then the DPS is also NP-hard.

Proof. Since the partially-relaxed-DPS is a relaxation of DPS, then DPS is at least as hard as the partially-relaxed-DPS. Consequently, if we prove that the partially-relaxedDPS is NP-hard, then the original DPS is also NP-hard.

Lemma 3. Any 3-SAT problem can be polynomially transformed into a corresponding partially-relaxed-DPS problem. 
Proof. Consider an arbitrary instance of the 3-SAT with clauses $C$ and variables $U$. We construct the corresponding partially-relaxed-DPS problem with $S=1$ and represented by graph $G=(V, E)$. Each $V^{t-1}$ and $V^{t}$ consists of $l+2 m$ vertices: the first $l$ vertices are representing the clauses $c_{h}$, the next $m$ vertices are representing the variables $u_{h}$ and the last $m$ vertices are representing the variables $\neg u_{h}$. The capacity of vertex $v_{i}^{t-1}$ representing clause $c_{h}$ is fixed to zero and the capacity of vertex $v_{i}^{t-1}$ representing variable $u_{h}$ or variable $\neg u_{h}$ is fixed to one. The capacity of vertex $v_{i}^{t}$ representing variable $u_{h}$ is fixed to one and the capacity of vertex $v_{i}^{t-1}$ representing variable $\neg u_{h}$ is fixed to zero. The capacity of vertex $v_{i}^{t}$ representing clause $c_{h}$ needs not be integer. The personnel wastage $\pi_{s i}^{t-1, t}$ and personnel recruitment $R_{i}^{t}$ are fixed to zero.

We define $E=E_{1} \cup E_{2} \cup E_{3}$. Let $E_{1}=\left\{\left(v_{l+i}^{t-1}, v_{l+i}^{t}\right) \mid i \in\{1, \ldots, m\}\right\}$, with $v_{l+i}^{t-1}$ and $v_{l+i}^{t}$ the vertices that are associated with variable $u_{i}$. Let $E_{2}=\left\{\left(v_{l+m+i}^{t-1}, v_{l+i}^{t}\right) \mid i \in\{1, \ldots, m\}\right\}$, with $v_{l+m+i}^{t-1}$ the vertex that is associated with variable $\neg u_{i}$ and $v_{l+i}^{t}$ the vertex that is associated with variable $u_{i}$. Let $L_{h}^{A}=\left\{j \in\{1, \ldots, l\} \mid\right.$ clause $c_{j}$ contains variable $\left.u_{h}\right\}$ and $L_{h}^{B}=\left\{j \in\{1, \ldots, l\} \mid\right.$ clause $c_{j}$ contains variable $\left.\neg u_{h}\right\}$. Let $E_{3}=\left\{\left(v_{l+i}^{t-1}, v_{j}^{t}\right) \mid i \in\right.$ $\left.\{1, \ldots, m\}, j \in L_{i}^{A}\right\} \cup\left\{\left(v_{l+m+i}^{t-1}, v_{j}^{t}\right) \mid i \in\{1, \ldots, m\}, j \in L_{i}^{B}\right\}$, with $v_{l+i}^{t-1}$ the vertex that is associated with variable $u_{i}, v_{l+m+i}^{t-1}$ the vertex that is associated variable $\neg u_{i}, v_{j}^{t}$ the vertex that is associated with clause $c_{j}$. All other edges that can be formed from $v_{i}^{t-1}$ to $v_{j}^{t}$ are considered to be fixed with $f_{i j}^{t-1, t}=0$. Personnel flow $f_{i j}^{t-1, t}$ of $E_{1}$ and $E_{2}$ is restricted to $\{0,1\}$ because of Eq. 18 and the integrality constraint of $f_{i j}^{t-1, t}$. Meanwhile, the integrality constraint personnel flow $f_{i j}^{t-1, t}$ of $E_{3}$ is not imposed.

The fuzzy triangular function of the desirability degree of vertex $v_{i}^{t}$ that is associated with variables $u_{i}$ and $\neg u_{i}$ is defined with $n_{i}^{L L}=-2, n_{i}^{d}=0$, and $n_{i}^{U L}=2$. The fuzzy triangular function of the desirability degree of vertex $v_{i}^{t}$ that is associated with clause $c_{h}$ is defined with $n_{h}^{L L}=0, n_{h}^{d}=\frac{2}{l}$, and $n_{h}^{U L}=6$. The fuzzy triangular function defining the promotion steadiness degree is relaxed by setting the lower and upper limit to -2 and 2 , and by fixin $\tilde{p}_{i j}$ and $n_{i}^{d}$ to zero.

The transformation of the 3-SAT problem to the partially-relaxed-DPS is clearly a polynomial transformation. It involves creating $2 l+4 m$ vertices for variable $u_{i}$ and clause 
$c_{h}$ in $V^{t-1}$ and $V^{t}$. Moreover, $(l+2 m)^{2}$ edges are created to accommodate personnel flows between two vertices.

Lemma 4. Any 3-SAT problem is satisfiable if and only if the corresponding partiallyrelaxed-DPS problem has an objective function value $\frac{1}{2}$.

Proof. The relationship of the partially-relaxed-DPS solution and the 3-SAT solution is as follows. A true value of variable $u_{i}$ corresponds to (i) zero personnel flow $f_{l+i, l+i}^{t-1, t}$ for $\left(v_{l+i}^{t-1}, v_{l+i}^{t}\right) \in E_{1}$ from vertex $v_{l+i}^{t-1} \in V^{t-1}$ that is associated with variable $u_{i}$ to vertex $v_{l+i}^{t} \in V^{t}$ that is associated with variable $u_{i}$, (ii) one personnel flow $f_{l+m+i, l+i}^{t-1, t}$ for $\left(v_{l+m+i}^{t-1}, v_{l+i}^{t}\right) \in E_{2}$ from vertex $v_{l+m+i}^{t-1} \in V^{t-1}$ that is associated with variable $\neg u_{i}$ to vertex $v_{l+i}^{t} \in V^{t}$ that is associated with variable $u_{i}$, and (iii) positive personnel flow $f_{l+i, j}^{t-1, t} \in\left[\frac{1}{l}, 1\right] \forall j \in L_{i}^{A}$ for $\left(v_{l+i}^{t-1}, v_{j}^{t}\right) \in E_{3}$ from vertex $v_{l+i}^{t-1} \in V^{t-1}$ that is associated with variable $u_{i}$ to vertex $v_{j}^{t} \in V^{t}$ that is associated with clause $c_{j}$. Meanwhile, a false value of variable $u_{i}$ corresponds to (i) one personnel flow $f_{l+i, l+i}^{t-1, t}$ for $\left(v_{l+i}^{t-1}, v_{l+i}^{t}\right) \in E_{1}$ from vertex $v_{l+i}^{t-1} \in V^{t-1}$ that is associated with variable $u_{i}$ to vertex $v_{l+i}^{t} \in V^{t}$ that is associated with variable $u_{i}$, (ii) zero personnel flow $f_{l+m+i, l+i}^{t-1, t}$ for $\left(v_{l+m+i}^{t-1}, v_{l+i}^{t}\right) \in E_{2}$ from vertex $v_{l+m+i}^{t-1} \in V^{t-1}$ that is associated with variable $\neg u_{i}$ to vertex $v_{l+i}^{t} \in V^{t}$ that is associated with variable $u_{i}$, and (iii) positive personnel flow $f_{l+m+i, j}^{t-1, t} \in\left[\frac{1}{l}, 1\right] \forall j \in L_{i}^{B}$ for $\left(v_{l+m+i}^{t-1}, v_{j}^{t}\right) \in E_{3}$ from vertex $v_{l+m+i}^{t-1} \in V^{t-1}$ that is associated with variable $\neg u_{i}$ to vertex $v_{j}^{t} \in V^{t}$ that is associated with clause $c_{j}$. Moreover, a true value of clause $c_{i}$ corresponds to the capacity of vertex $v_{i}^{t} \in V^{t}$ that is associated with clause $c_{i}$ to be $\left[\frac{1}{l}, 3\right]$.

We prove that any 3-SAT problem is satisfiable if and only if the corresponding partially-relaxed-DPS problem has an objective function value $\frac{1}{2}$. Suppose that the 3 -SAT problem is satisfiable. Then a truth assignment $U$ corresponds to $f_{i j}^{t-1, t}$ values that satisfy Eqs. 18 19. The capacity of vertex $v_{i}^{t} \in V^{t}$ that is associated with a true value of variable $u_{i}$ or $\neg u_{i}$ is one, and consequently the corresponding desirability degree is $\frac{1}{2}$. Meanwhile, the capacity of vertex $v_{i}^{t} \in V^{t}$ that is associated with a false value of variable $u_{i}$ or $\neg u_{i}$ is one, and therefore the corresponding desirability degree is 1. The capacity of vertex $v_{i}^{t} \in V^{t}$ that is associated with clause $c_{h}$ is in $\left[\frac{1}{l}, 3\right]$, thus it leads to the corresponding desirability degree $\in\left[\frac{1}{2}, 1\right]$. The promotion steadiness degree is always an element of $\left[\frac{1}{2}, 1\right]$ 
because the personnel flow is bounded to $[0,1]$. Therefore, the $\kappa\left(n^{t}, F^{t-1, t}\right)$ value will be $\frac{1}{2}$.

Now, suppose that the objective function of the corresponding partially-relaxed-DPS problem is $\kappa\left(n^{t}, F^{t-1, t}\right)=\frac{1}{2}$. The capacity of vertex $v_{i}^{t} \in V^{t}$ that is associated with clause $c_{h}$ is $\in\left[\frac{1}{l}, 3\right]$. Therefore, all the clauses $c_{h}$ in $C$ are true and the 3 -SAT problem is satisfiable.

Now, we prove the main theorem of the complexity of DPS.

Theorem 1. DPS is NP-hard.

Proof. Based on Lemma 1, 3 and 4, the corresponding partially-relaxed-DPS is in NP and is at least as hard as the 3-SAT problem. Therefore, the corresponding partiallyrelaxed-DPS is an NP-hard problem. Then, based on Lemma 2, the DPS problem is also NP-hard.

Consider a special case of the DPS problem as follows:

Definition 3. A relaxed-DPS is a relaxation of DPS in which the integrality constraint of the variables $n_{s i}^{t}, f_{\text {sij }}^{t-1, t}$, and $R_{i}^{t}$ is not imposed.

Lemma 5. The relaxed-DPS problem is in $P$.

Proof. The relaxed-DPS problem can be modeled as a linear programming model. In the relaxed-DPS, $n_{i}^{t, s}, f_{i j}^{t-1, t, s}, R_{i}^{t}$ are elements of $\mathbb{R}^{+}$. According to Hannan (1981), the triangular fuzzy membership functions can be modeled as linear constraints and continuous variables. In this way, the binary variables $q_{i L}^{s b 1}, q_{i R}^{s b 1}, q_{i j L}^{s b 2}, q_{i j R}^{s b 2}$ can be omitted from the model. Since the DPS problem only involves fuzzy triangular membership functions, the transformation using Hannan (1981)'s method is a polynomial transformation. Each fuzzy triangular membership function can be modeled as a linear constraint with 7 decision variables. Thus, the fuzzy triangular membership functions are modeled using $k(k+1)$ constraints and $7 k(k+1)$ variables.

As the relaxed-DPS problem can be modeled as a linear programming model, and there exists a polynomial algorithm to solve linear programming models (Khachian, 1979), the relaxed-DPS is in $\mathrm{P}$. 
Based on Lemma 5, the relaxed-DPS is in P, thus there exists an efficient method to solve the DPS without the integrality constraints of $n_{s i}^{t}, f_{s i j}^{t-1, t}, R_{i}^{t}$. The overall degree of the relaxed-DPS gives an approximation for the value $\kappa\left(\mathbf{n}^{\mathbf{t}}, \mathbf{F}^{\mathbf{t}-\mathbf{1}, \mathbf{t}}\right)$ of the DPS problem.

\section{Practical application and illustration}

\subsection{Model application in practice}

A lot of previous work suggests decision support methods to attain a desired personnel structure under control by recruitment. However, not every desired personnel structure is attainable under recruitment control only (Guerry, 1993; Davies, 1982). In that case, the DPS model can be used to find an optimal personnel strategy for partially stochastic manpower systems. The DPS model assumes that both recruitment and promotion strategies are controlled by management, but that the resulting promotion flows and personnel structures should be as close as possible to their preferred values.

In practice, personnel decisions often need to anticipate to uncertain events in future. In order to prevent future personnel shortages caused by wastage, manpower planners proactively need to decide on their personnel strategies (De Feyter, 2007). Since recruitment and selection procedures can take a significant amount of time, it is reasonable to assume that the recruitment strategy should be chosen before the exact wastage is known. Therefore, the DPS model treats wastage as a stochastic variable. In the context of uncertainty, it is common practice to consider a finite number of scenarios Shapiro et al., 2009). The DPS model evaluates personnel strategies for a set of possible wastage scenarios. Therefore, the practitioner can rely on prior work that proposes several probability distributions and corresponding parameter estimators to model wastage (e.g. Ugwuowo and McClean (2000); De Feyter (2006)). A probability distribution of wastage can be approximated at the beginning of a planning period. From this distribution, one can generate a finite number of wastage scenarios. Subsequently, the DPS model calculates the optimal recruitment vector. The input for the model is based on the initial 
personnel structure, the selected possible wastage scenarios and the specification of the desired personnel structure and promotion flows and their corresponding lower and upper bounds. This allows the practitioner to process the recruitment and selection procedures so that the necessary personnel is available in time. At the end of the planning period, the actual personnel wastage and the resulting personnel structure after recruitment are known. This allows reassessing the promotion strategy by optimizing both the degree of desirability and degree of promotion steadiness. Therefore, the practitioner can use the deterministic approach to balance the degree of promotion steadiness and desirability (Section 2), in which the wastage is known and the recruitment strategy is no longer a decision variable. This procedure results in a good balance of the desirability degree and the promotion steadiness degree.

\subsection{Computational example}

This section provides an illustration that gives an overview of the methodology to the reader. This illustration gives an overview of the methodology to the reader. The model was solved with CPLEX 12.4, 4 threads, maximum 2 GB memory and 600 seconds time limit. The experiment was conducted using an Intel core i7 2.7 GHz, 6 GB memory and a Windows operating system.

Assume a manpower system with $k=4$ subgroups, initial personnel structure $\mathbf{n}^{\mathbf{t}-\mathbf{1}}=$ [357 10591447 ], the upper bound of the total number of personnel $N^{\max }=1000$, desired personnel structure $\mathbf{n}^{\mathbf{d}}=\left[\begin{array}{l}3448587484\end{array}\right]$, and internal transition matrix

$$
\mathbf{P}=\left[\begin{array}{llll}
0.72 & 0.03 & 0.05 & 0.04 \\
0.00 & 0.84 & 0.01 & 0.02 \\
0.12 & 0.04 & 0.73 & 0.04 \\
0.00 & 0.09 & 0.03 & 0.81
\end{array}\right]
$$

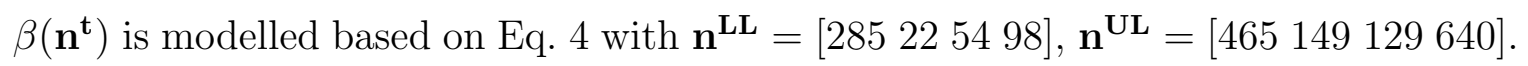
$\gamma\left(f_{i j}^{t-1, t}\right)$ is modelled based on Eq. 6 with $\mathbf{P}^{\mathbf{L L}}$ and $\mathbf{P}^{\mathbf{U L}}$ as follows: 


\section{$\gamma_{\mathrm{ij}}\left(\mathrm{f}_{\mathrm{ij}}^{\mathrm{t}-1, \mathrm{t}}\right)$ triangular function}

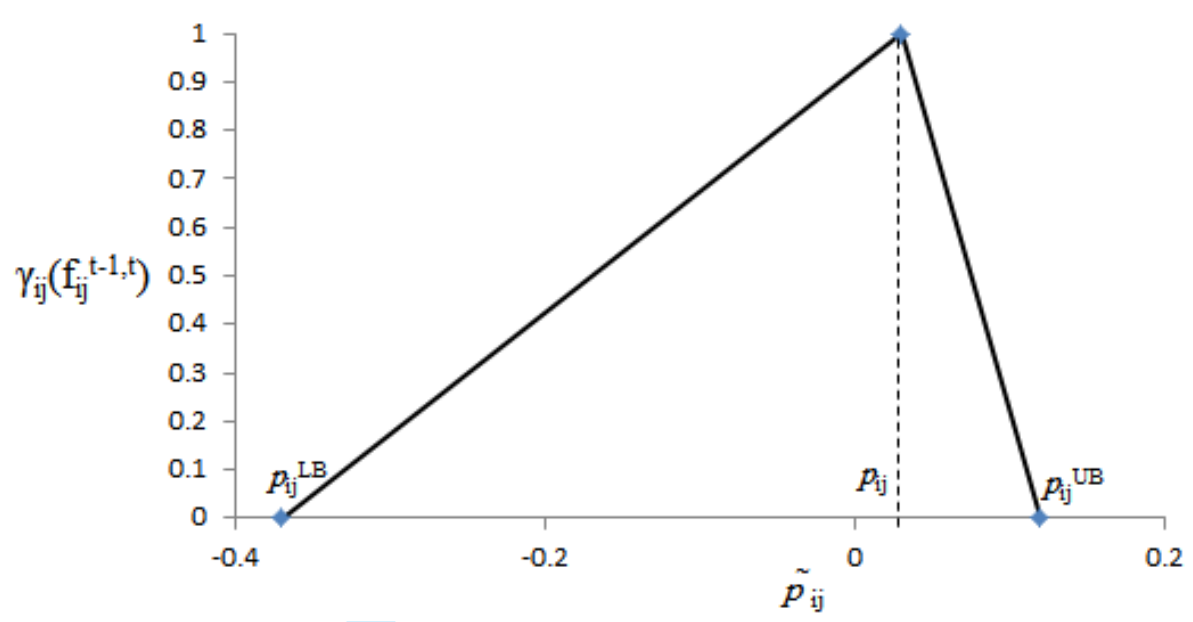

Figure 1: A graphical presentation of the triangular membership function

$$
\mathbf{P}^{\mathbf{L L}}=\left[\begin{array}{rrrr}
0.62 & -0.37 & -0.17 & -0.1 \\
-0.2 & 0.78 & -0.14 & -0.07 \\
-0.16 & -0.06 & 0.41 & -0.01 \\
-0.39 & -0.29 & -0.35 & 0.64
\end{array}\right] \mathbf{P}^{\mathrm{UL}}=\left[\begin{array}{cccc}
1.06 & 0.12 & 0.21 & 0.13 \\
0.13 & 1.14 & 0.36 & 0.11 \\
0.14 & 0.24 & 1.01 & 0.34 \\
0.09 & 0.43 & 0.39 & 1.03
\end{array}\right]
$$

It should be noted that $\mathbf{P}^{\mathbf{L L}}$ and $\mathbf{P}^{\mathbf{U L}}$ are not transition matrices. They contain the lower limit $p_{i j}^{L L}$ and upper limit $p_{i j}^{U L}$ to construct the triangular function for $\tilde{p}_{i j}$ in Eq. 6. However, the proportion of the performed personnel flows is always in [0,1]. For example, for $i=1$ and $j=2$, the fuzzy triangular function for $\gamma_{12}\left(f_{12}^{t-1, t}\right)$ is represented in Fig. 1.

The wastage proportion $w_{i}$ is assumed to follow a normal distribution with mean $\bar{w}_{i}$ and standard deviation $w_{i}^{e}$. Let $\overline{\mathbf{w}}=\left[\begin{array}{lllll}0.16 & 0.13 & 0.07 & 0.07\end{array}\right]$ and $\mathbf{w}^{\mathbf{e}}=\left[\begin{array}{llll}0.01 & 0.01 & 0.01 & 0.01\end{array}\right]$.

We compare the deterministic and stochastic approaches:

- Considering the deterministic case $\mathbf{w}=\overline{\mathbf{w}}$, the CPLEX solver produces an overall degree $\kappa\left(\mathbf{n}^{\mathbf{t}}, \mathbf{F}^{\mathbf{t}-\mathbf{1 , t}}\right)=0.80852$ with recruitment vector $\mathbf{R}^{\mathbf{t}}=\left[\begin{array}{llll}77 & 0 & 0 & 0\end{array}\right]$. However, if the recruitment vector $\mathbf{R}^{\mathbf{t}}=\left[\begin{array}{llll}77 & 0 & 0 & 0\end{array}\right]$ is tested under stochastic case $\mathbf{w}, \bar{\kappa}=0.78099$.

- Assuming that in the stochastic case $w_{i}$ follows a normal distribution, 1000 scenarios 
were generated to approximate $w$. The CPLEX solver produces an overall degree $\bar{\kappa}=0.8019$ with recruitment vector $\mathbf{R}^{\mathbf{t}}=\left[\begin{array}{llll}55 & 0 & 0 & 13\end{array}\right]$.

- Approximating $\bar{\kappa}$ using the relaxed-DPS (the integrality constraint of the variables $n_{s i}^{t}, f_{s i j}^{t-1, t}$, and $R_{i}^{t}$ is not imposed), the CPLEX solver produces an overall degree $\kappa\left(\mathbf{n}^{\mathbf{t}}, \mathbf{F}^{\mathbf{t}-\mathbf{1 , t}}\right)=0.81095$ with recruitment vector $\mathbf{R}^{\mathbf{t}}=\left[\begin{array}{l}45.5480 \\ 0\end{array}\right.$ 41.452]. However, if the integrality constraints are imposed with recruitment vector $\mathbf{R}^{\mathbf{t}}=\left[\begin{array}{llll}46 & 0 & 0 & 41\end{array}\right]$, $\bar{\kappa}=0.79976$.

The stochastic approach yields a better $\kappa\left(\mathbf{n}^{\mathbf{t}}, \mathbf{F}^{\mathbf{t}-\mathbf{1}, \mathbf{t}}\right)$ since it considers all 1000 scenarios whereas the deterministic approach only considers the average of the wastage probabilities. In other words, the stochastic approach does not only consider the measure of center tendency of the wastage but it also considers statistical dispersion of the wastage. As larger the statistical dispersion of the wastage, as more accurate the stochastic approach. Moreover, we can observe that the recruitment vectors of the two approaches differ significantly. The stochastic approach results in a recruitment vector that is able to deal with different scenarios. Instead of only recruiting the personnel into subgroup 1, the stochastic approach suggests that personnel should be recruited into subgroups 1 and 4 .

In Figures 2,4, we compare two approaches, i.e. (a) the stochastic and (b) the relaxedDPS. Clearly, the stochastic approach is the most suited approach to deal with the DPS problem. In three figures, the medians of the stochastic approach are at least as good as the relaxed DPS. The relaxed DPS, in several scenarios, results in very low values of desirability and promotion steadiness degree.

\section{Conclusions and further research}

In the present paper, we successfully provided a model and approach to the trade-off problem between the degree of desirability and the degree of promotion steadiness. The model has the advantage that it fulfills the personnel requirements as good as possible, while maintaining the promotion strategy as much as possible. This way, one can obtain 


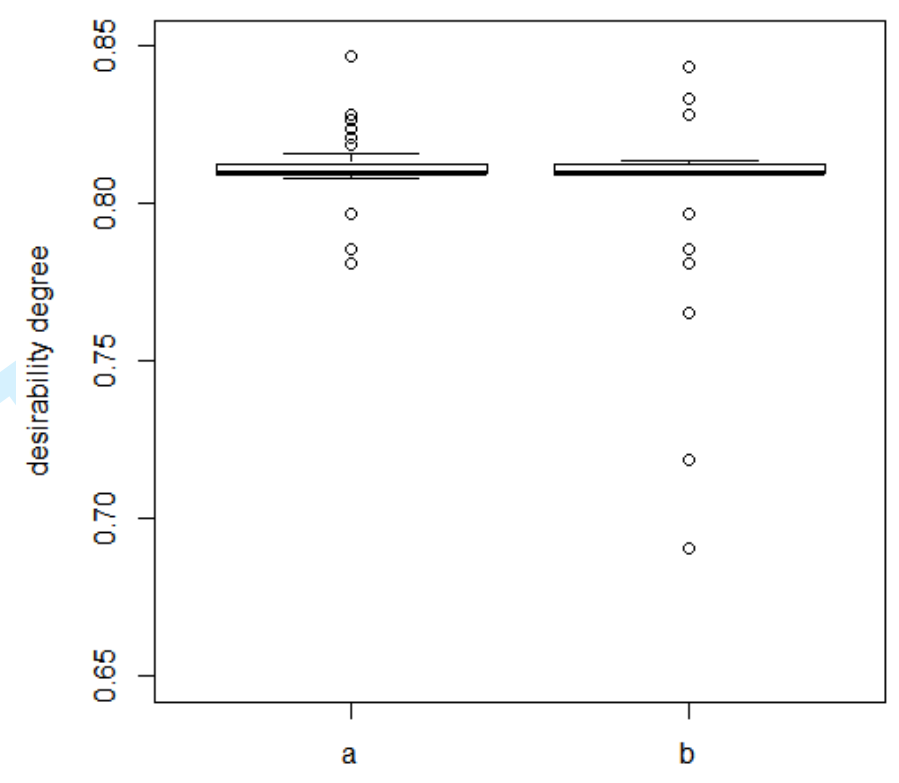

Figure 2: The desirability degree obtained by the (a) stochastic, (b) relaxed DPS

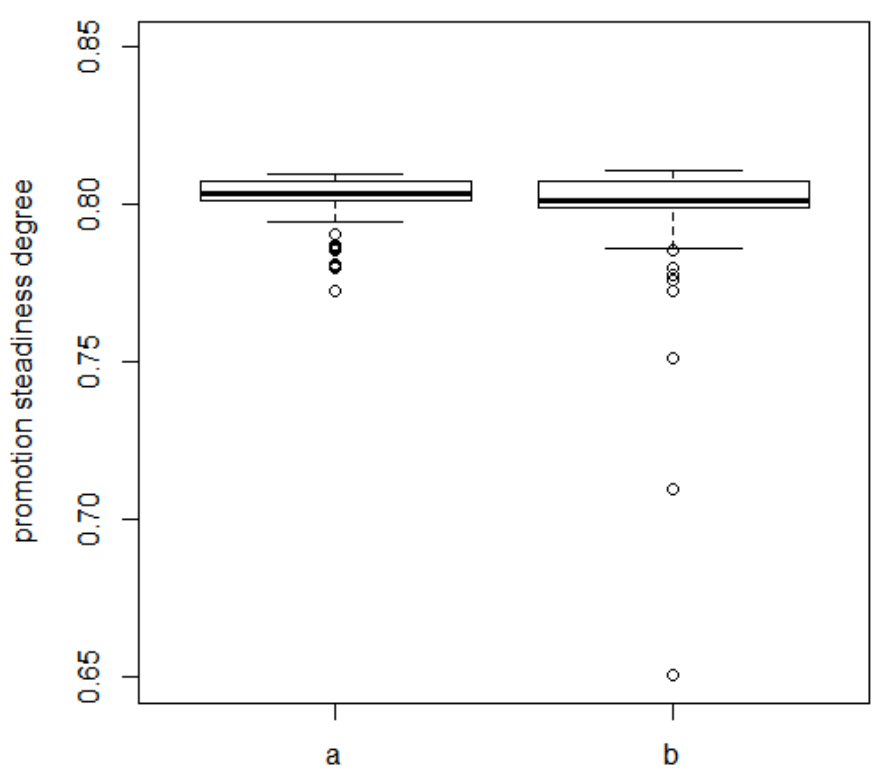

Figure 3: the promotion steadiness degree obtained by the (a) stochastic, (b) relaxed DPS 


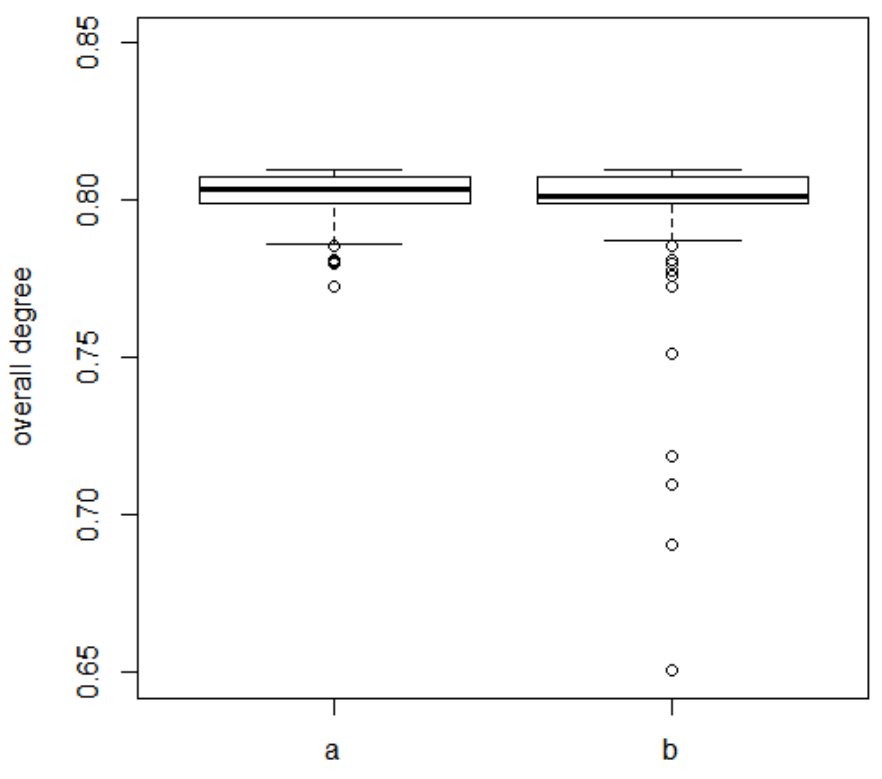

Figure 4: The overall degree obtained by the (a) stochastic, (b) relaxed DPS

a good balance between the organizational personnel needs to support its long-term strategy and the employees? satisfaction regarding their career opportunities. Further, our model also deals with the randomness of the wastage probability. It provides a robust recruitment vector that can anticipate future actual wastage.

Although the methodology presented in this paper makes a valuable contribution to the field, we need to consider some limitations and questions which further research could address.

Firstly, as in previous work on control and optimization in manpower planning (Dimitriou et al., 2013; De Feyter and Guerry, 2009), we integrated fuzzy set theory in our model. We used a fuzzy triangular function to express the degree of desirability and the degree of promotion steadiness. However, future work can consider other fuzzy membership functions. More suited fuzzy membership functions may be needed to describe the opinion of the decision makers in formulating the degree of desirability and the degree of promotion steadiness.

Secondly, our illustration presents a problem instance with only a restricted number 
of subgroups. In practice, however, it is often necessary to partition the personnel system in a considerable higher number of homogeneous subgroups. This is necessary to enable modeling the heterogeneity in personnel behavior (Ugwuowo and McClean, 2000; De Feyter, 2006)). In Section 3, we showed that the DPS problem is NP-hard. Consequently, an increase in the number of subgroups may lead to an exponential increase in computing time. A decomposition based MIP approach may be needed to solve problem instances with high numbers of subgroups. Further research could also focus on the specific characteristics of the personnel system for which the DPS problem is not NPhard. For these DPS problems, future work can search for polynomial algorithms. We already showed that the relaxed DPS-problem is not NP-hard. In most previous work in manpower planning, the integrality constraints on the personnel structure, recruitments and promotions flows are indeed neglected (Guerry, 2008). However, this obviously is not a realistic assumption in real-world applications. Nevertheless, in future research, it can be investigated whether or not the solution of the relaxed DPS-problem is a good approximation for the solution of the DPS problem.

Thirdly, future work could refine our model to include real world requirements. Some large organizations divide their operations into several divisions at different locations. Planning the recruitment, the promotion as well as the transfers between different division and/or locations are beneficial to these type of organizations (Guerry and De Feyter, 2012; Dimitriou et al., 2013). Furthermore, our model assumes that sufficient personnel is always available to be recruited. However, this assumption may not hold when a certain subgroup requires specific skills and qualifications. We could also assume uncertainty in the desired personnel structure when the work demand is uncertain, as considered in Zhu and Sherali (2009). Additionally, the present model does not include financial consequences, which are very important factors in determining a manpower planning strategy for commercial organizations. Finally, the presented model deals with a time frame of one period (from $t-1$ to $t$ ). Future research could extend our model by including multi-period time horizon. 


\section{References}

G. K. Agrafiotis. A grade-specific stochastic model for the analysis of wastage in manpower systems. The Journal of the Operational Research Society, 35(6):549-554, 1984.

David J. Bartholomew, Andrew F. Forbes, and Sally I. McClean. Statistical techniques for manpower planning. John Wiley \& Sons, 1991.

Asis Kumar Chattopadhyay and Arindam Gupta. A stochastic manpower planning model under varying class sizes. Annals of Operations Research, 155(1):41-49, July 2007.

G. S. Davies. Control of grade sizes in partially stochastic markov manpower model. Journal of Applied Probability, 19:439-443, 1982.

Tim De Feyter. Modelling heterogeneity in manpower planning : dividing the personnel system into more homogeneous subgroups. Applied Stochastic Models in Business and Industry, 22(March):321-334, 2006.

Tim De Feyter. Modeling mixed push and pull promotion flows in Manpower Planning. Annals of Operations Research, 155(1):25-39, July 2007.

Tim De Feyter and Marie-Anne Guerry. Evaluating Recruitment Strategies Using Fuzzy Set Theory in Stochastic Manpower Planning. Stochastic Analysis and Applications, 27(6):1148-1162, 2009.

V.A. Dimitriou, A.C. Georgiou, and N. Tsantas. The multivariate non-homogeneous markov manpower system in a departmental mobility framework. European Journal Of Operational Research, 228:112-121, 2013.

Michael R. Garey and David S. Johnson. Computers and Intractability - A Guide to the Theory of NP-Completeness. W. H. Freeman and Company, 1979.

Marie-Anne Guerry. The probability of attaining a structure in a partially stochastic model. Advances in applied probability, 25(4):818-824, 1993. 
Marie-Anne Guerry. On the evolution of stock vectors in a deterministic integer-valued markov system. Linear Algebra and its Applications, 429:1944-1953, 2008.

Marie-Anne Guerry and Tim De Feyter. Optimal recruitment strategies in a multi-level manpower planning model. Journal of the Operational Research Society, 63(7):931-940, 2012.

Edward L. Hannan. Linear programming with multiple fuzzy goals. Fuzzy Sets and Systems, 6:235-248, 1981.

L. G. Khachian. A polynomial algorithm in linear programming. Soviet Mathematics Doklady, 20:191-194, 1979.

Ute-Christine Klehe, Jelena Zikic, Annelies E.M. Van Vianen, and Irene E. De Pater. Career adaptability, turnover and loyalty during organizational downsizing. Journal of Vocational Behavior, 79(1):217-229, 2011.

K. Nilakantan, J.K. Sankaran, and BG Raghavendra. A proportionality model of Markov manpower systems. Journal of Modelling in Management, 6(1):100-122, 2011.

Alexander Shapiro, Darinka Dentcheva, and Andrzej Ruszczyński. Lectures on Stochastic Programming Modeling and Theory. SIAM, 2009.

Milind G. Sohoni, Ellis L. Johnson, and T. Glenn Bailey. Long-Range Reserve Crew Manpower Planning. Management Science, 50(6):724-739, June 2004.

Haiqing Song and Huei-Chuen Huang. A successive convex approximation method for multistage workforce capacity planning problem with turnover. European Journal of Operational Research, 188(1):29-48, 2008.

F.I. Ugwuowo and S.I. McClean. Modelling heterogeneity in a manpower system: a review. Applied Stochastic Models in Business and Industry, 16(2):2, 2000.

X Zhu and H D Sherali. Two-stage workforce planning under demand fluctuations and uncertainty. Journal of the Operational Research Society, 60:94-103, 2009. 
$\gamma_{\mathrm{ij}}\left(\mathrm{f}_{\mathrm{ij}}^{\mathrm{t}-1, \mathrm{t}}\right)$ triangular function

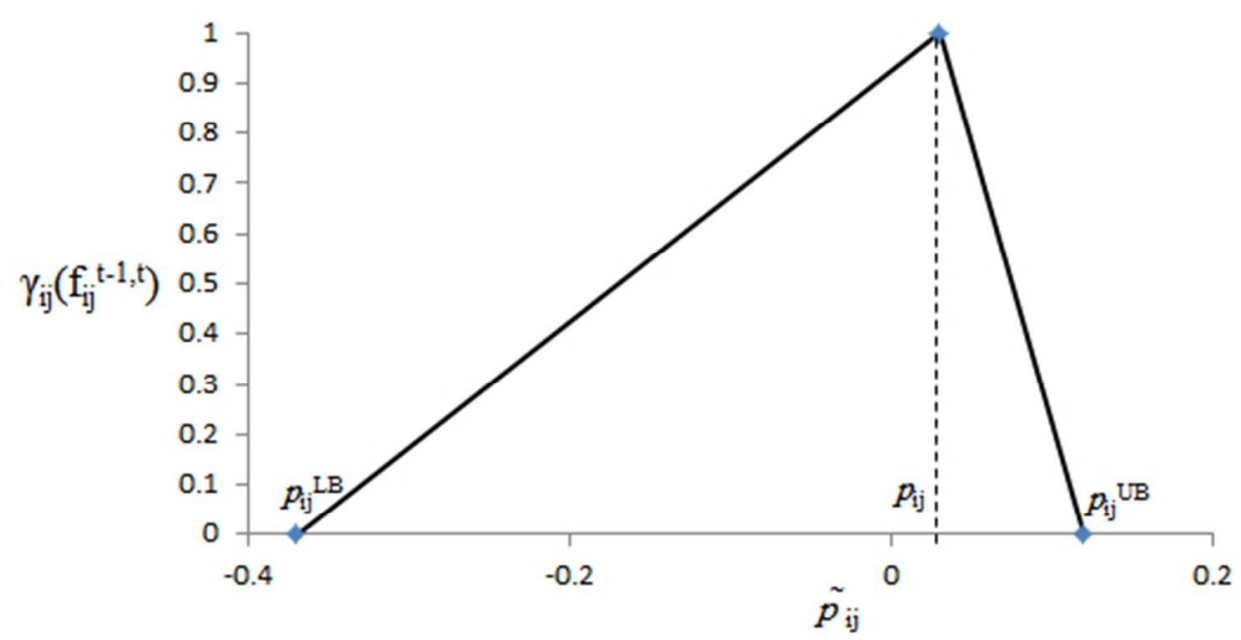

A graphical presentation of the triangular membership function $145 \times 87 \mathrm{~mm}(96 \times 96$ DPI) 


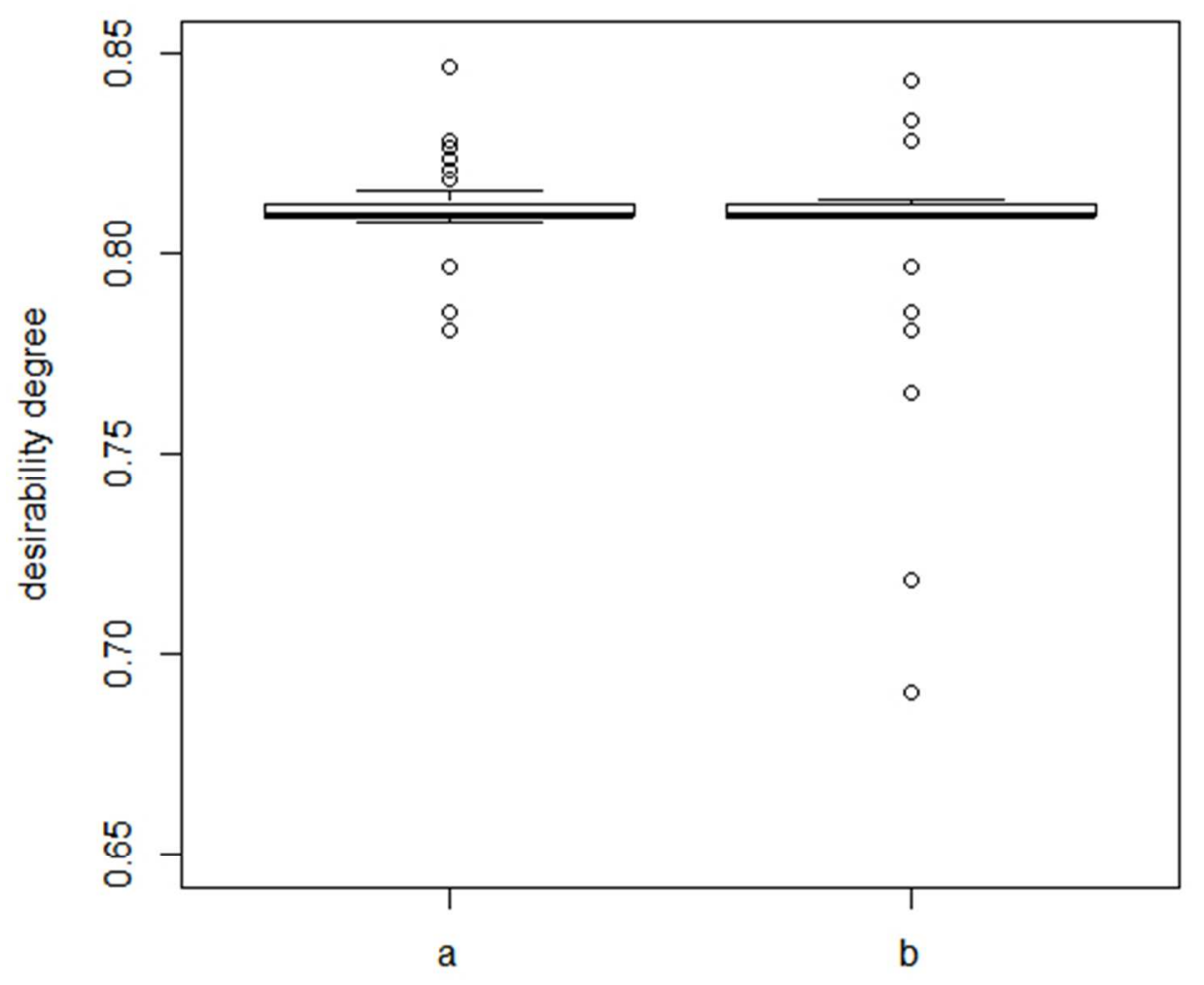

The desirability degree obtained by the (a) stochastic, (b) relaxed DPS $198 \times 197 \mathrm{~mm}(72 \times 72$ DPI $)$ 
1

2

3

4

5

6

7

8

9

10

11

12

13

14

15

16

17

18

19

20

21

22

23

24

25

26

27

28

29

30

31

32

33

34

35

36

37

38

39

40

41

42

43

44

45

46

47

48

49

50

51

52

53

54

55

56

57

58

59

60

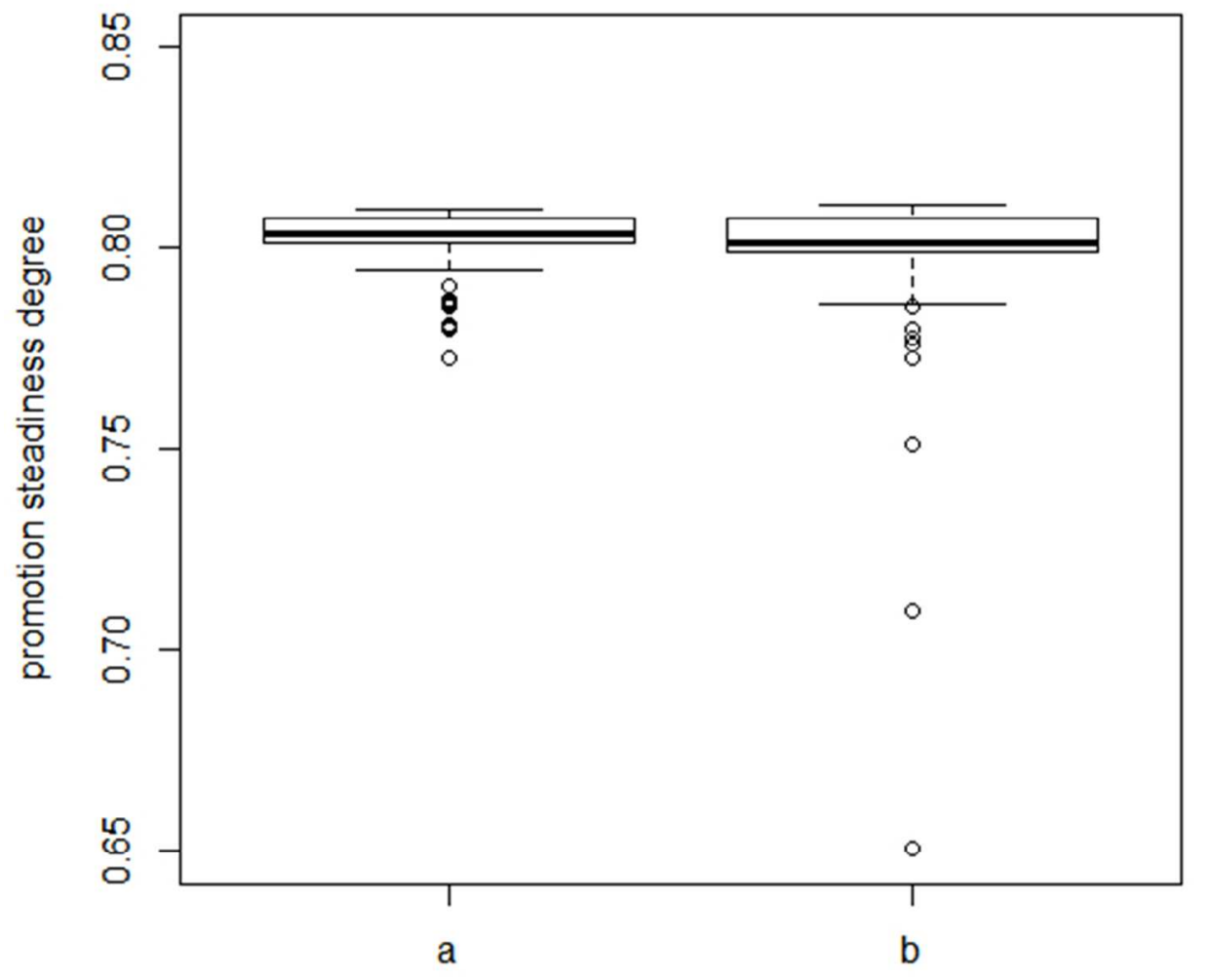

The promotion steadiness degree obtained by the (a) stochastic, (b) relaxed DPS $198 \times 197 \mathrm{~mm}(72 \times 72 \mathrm{DPI})$ 


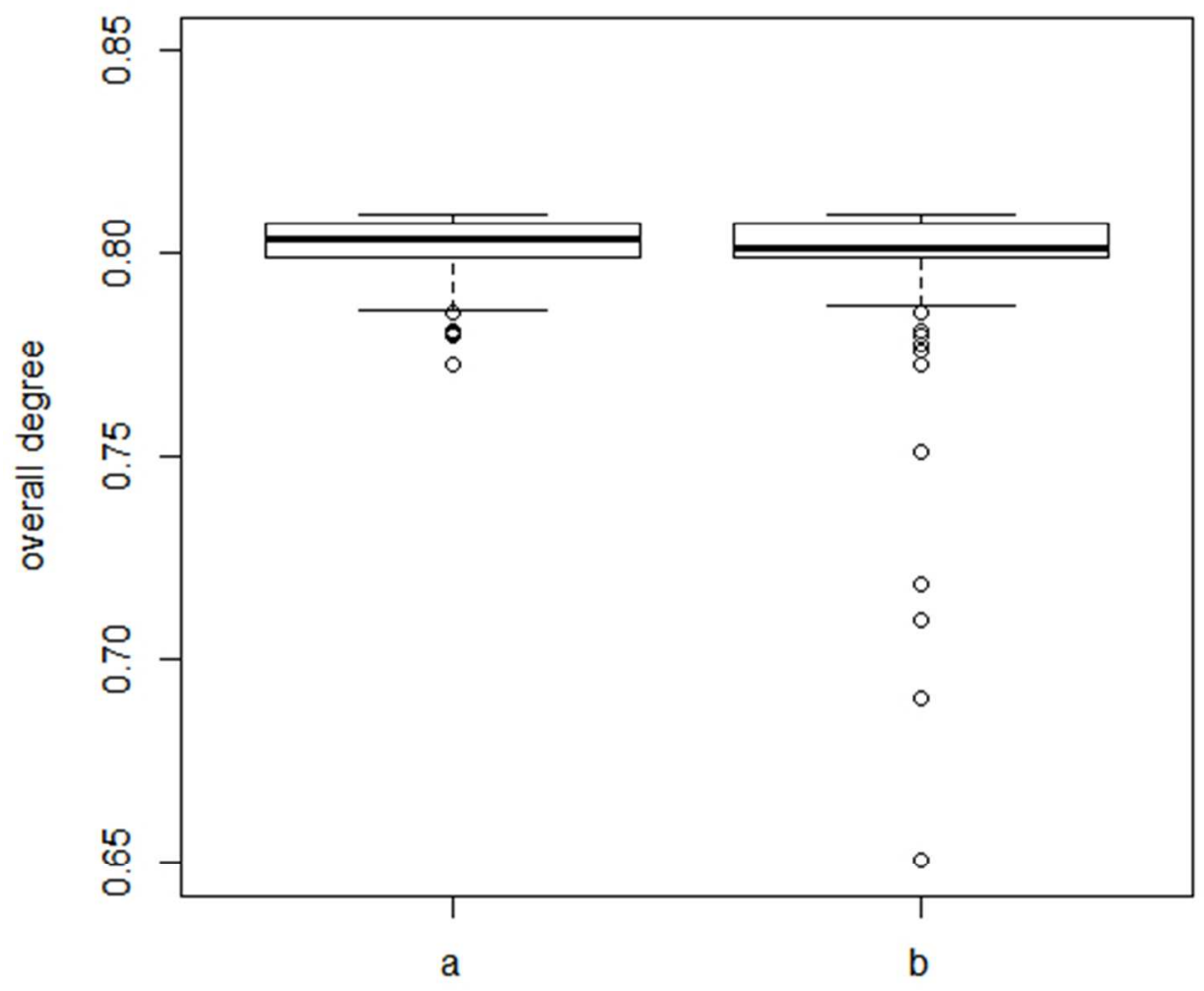

The overall degree obtained by the (a) stochastic, (b) relaxed DPS $198 \times 197 \mathrm{~mm}(72 \times 72 \mathrm{DPI})$ 